\title{
High GADA titer increases the risk of insulin requirement in LADA patients: a 7-year follow-up (NIRAD study 7)
}

\author{
Simona Zampetti, Giuseppe Campagna, Claudio Tiberti, Marco Songini ${ }^{1}$, \\ Maria Luisa Arpi ${ }^{2}$, Giuseppina De Simone ${ }^{3}$, Efisio Cossu', Lorenzo Cocco5, \\ John Osborn ${ }^{6}$, Emanuele Bosi ${ }^{7}$, Francesco Giorgino ${ }^{8}$, Marialuisa Spoletini and \\ Raffaella Buzzetti on behalf of the NIRAD Study Group*
}

Department of Experimental Medicine, 'Sapienza' University of Rome, Viale Regina Elena 324, 00161 Rome, Italy, ${ }^{1}$ Department of Internal Medicine, S. Michele Hospital, Cagliari, Italy, ${ }^{2}$ Endocrinology, Garibaldi di Nesima Hospital, Catania University, Catania, Italy, ${ }^{3}$ Diabetologic Center, Distretto 54 ASL Napoli 3, Naples, Italy, ${ }^{4}$ Endocrinology and Diabetes, Department of Medical Sciences, University of Cagliari, Cagliari, Italy, ${ }^{5}$ U.O.C. Diabetologia/ Endocrinology, 'A. Cardarelli' Hospital, Campobasso, Italy, ${ }^{6}$ Department of Public Health Sciences and Infectious Diseases, 'Sapienza' University of Rome, Rome, Italy, ${ }^{7}$ San Raffaele Scientific Institute, Diabetes Research Institute, Milan, Italy and ${ }^{8}$ Section of Internal Medicine, Endocrinology, Andrology and Metabolic Disease, Department of Emergency and Organ Transplantation, University of Bari, School of Medicine, Bari, Italy *(Details of the NIRAD Study group is presented in the Acknowledgements section)

\author{
Correspondence \\ should be addressed \\ to R Buzzetti \\ Email \\ raffaella.buzzetti@ \\ uniroma1.it
}

\begin{abstract}
Objective: The aim of this study was to determine whether glutamic acid decarboxylase antibody (GADA) titer and other clinical parameters could define the risk of progression to insulin therapy in latent autoimmune diabetes in adults (LADA) patients during a 7-year follow-up.

Methods: This study involved 220 LADA and 430 type 2 diabetes subjects followed up for 7 years from the time of GADA screening to evaluate their progression toward insulin therapy. Kaplan-Meier curves and multivariate logistic regression analysis were performed to identify the markers capable of influencing this progression.

Results: During the follow-up, the drop out was $4 \%$ in both groups. A total of $119(56.1 \%)$ out of 212 LADA patients required insulin during the 7 years of follow-up. The Kaplan-Meier plots showed that 74/104 (71.1\%) of high GADA titer required insulin compared with 45/108 (41.6\%) of low GADA titer and with 86/412 (20.9\%) of type 2 diabetes ( $P<0.0001$ for both). A BMI of $\leq 25 \mathrm{~kg} / \mathrm{m}^{2}$ and IA-2 IC and zinc transporter 8 (ZnT8) positivity were also shown as the markers of faster progression $(P<0.0001$ for both). The proportion of LADA patients requiring insulin was significantly higher in the group of subjects treated also with sulfonylurea in the first year from diagnosis compared with those treated with diet and/or insulin sensitizers $(P<0.001)$. The multivariate analysis confirmed that the presence of high GADA titer was a significant predictor of insulin requirement $(P<0.0001$, OR $=6.95)$.

Conclusions: High GADA titer, BMI $\leq 25, \mathrm{ZnT} 8$ and IA-2 $\mathrm{IC}$ positivity and sulfonylurea treatment, in the first year from diagnosis, significantly increase the progression toward insulin requirement in LADA patients.
\end{abstract}

European Journal of Endocrinology

(2014) 171, 697-704

\section{Introduction}

Latent autoimmune diabetes in adults (LADA) is a form of autoimmune-mediated diabetes, usually diagnosed by the presence of islet autoantibodies, namely glutamic acid decarboxylase antibodies (GADAs), with or without protein tyrosine phosphatase IA-2 antibodies (IA-2As) $(1,2)$ and/or zinc transporter 8 (ZnT8) (3). (c) 2014 European Society of Endocrinology Printed in Great Britain
Published by Bioscientifica Ltd. 
Although LADA subjects are characterized by the presence of specific antibodies for autoimmune diabetes, they do not require insulin therapy initially (4). During the early phase of disease, individuals with LADA can retain good metabolic control through diet alone or by taking oral hypoglycemic drugs, and this state can last from months to years. However, a percentage of LADA patients progress toward insulin requirement, indicating that LADA involves a slow and progressive loss of $\beta$-cells (5), although the factors influencing this progression are not completely known (6). The first evidence, demonstrating that a high GADA titer is a stronger predictor of insulin requirement than a general GADA positivity, was obtained from two studies with a limited number of LADA patients $(7,8)$. UKPDS 25 reported, in a larger population of LADA patients, that high GADA titer was associated with a significant higher risk of insulin requirement only among subjects older than 55 years at diagnosis (1). Afterwards, conflicting results were reported in the literature; some studies demonstrated that a high titer of GADA was associated with a shorter insulin-free period $(9,10,11)$, whereas others did not support this hypothesis $(6,12)$.

In a previous study, we demonstrated the presence of two different populations among individuals with LADA; analysis of GADA titer showed a bimodal distribution that identified two subgroups of subjects with either a low or a high GADA titer (13).

Assuming that the more prominent features of insulin deficiency, that characterize high GADA titer compared with low GADA titer (13), define a more reduced $\beta$-cell function, it may follow that patients with high GADA titer would require insulin therapy earlier, compared with those with low GADA titer.

The aim of the present prospective study was to determine whether GADA titer would affect progression to insulin therapy in LADA patients during a 7-years of follow-up. We also looked for other biochemical and clinical parameters associated with early development of insulin requirement.

\section{Subjects and methods}

\section{Study design and populations}

In the non-insulin requiring autoimmune diabetes (NIRAD) study, we screened 5330 type 2 diabetes patients from 83 diabetes centers equally distributed in the entire mainland and island of Italian territory. A total of 250 subjects of 5330 type 2 diabetes patients were positive for GADA (4.5\%) and thus we defined these patients as having
LADA. Out of 250 LADA patients, 30 patients did not give their consent to be followed-up in the NIRAD study but only to participate in the initial screening. A total of 220 LADA patients were followed up for 7 years in this study: 430 out of 5080 type 2 diabetes patients, matched for gender and duration of disease with LADA patients, were selected as control group. A total of 220 LADA patients, mean age at onset $50.6 \pm 13.1$ years, and 430 GADA negative type 2 diabetes subjects, matched for age and gender, mean age at onset $51.2 \pm 10.5$ years, from the NIRAD study cohort of 5330 consecutive cases of type 2 diabetes subjects, recruited between February 2001 and January $2006(13,14)$, were evaluated in this study.

Inclusion criteria for the NIRAD study were as follows: i) diagnosis of diabetes according to the American Diabetes Association (15); ii) no insulin requirement and no evidence of ketosis from diagnosis to screening time; and iii) disease duration between 6 months and 5 years. The exclusion criteria included prior insulin therapy, pregnancy, and the presence of any other severe disease.

Sixty per cent of LADA subjects identified in the NIRAD study had a disease duration $<2$ years at the time of antibody screening for GAD.

\section{Antibodies measurement}

At the time of GADA screening, the clinical and biochemical characteristics and antibodies (IA-2 IC) were evaluated $(13,14)$.

GADAs and IA-2As were measured centrally at San Raffaele Hospital in Milan and at 'Sapienza' University in Rome, respectively, using a radiobinding assay with in vitro-translated [35S] methionine-labeled GAD65 and IA-2 IC (amino acids 605-979) $(13,16)$. All samples were tested in duplicate.

The following results were obtained for these GADA and IA-2A assays at the Diabetes Antibody Standardization Program (DASP) between 2002 and 2005: GADA sensitivity 84,86 , and $88 \%$; GADA specificity 97,97 , and $92 \%$ and IA-2A sensitivity 60,62 , and $70 \%$ and IA-2A specificity 100, 99, and 99\% (13).

The results for GADAs were converted into arbitrary units by extrapolation from a standard curve with a local standard designated 100 arbitrary units. The thresholds for positivity were determined from the 99th centile of control subjects and corresponded to three arbitrary units for GADAs.

The distribution of GADA titer in patients with LADA was independent of diabetes duration and showed a bimodal distribution. Consistently with this 
observation, patients with LADA (GADA titer $>3$ arbitrary units) were divided into subgroups representing the two distributions, namely low (being $\leq 32$ units) and high (being $>32$ units) GADA titers (13). Based on DASP as a reference (17) the threshold of 32 units was equivalent to $300 \mathrm{WHO}$ units (13).

The samples with low GADA titer were validated for glutamic acid decarboxylase (GAD)-specific binding by competition assay with excess of cold GAD (13).

The sera of LADA patients were re-analyzed and confirmed as GADA positive in a second Italian reference laboratory (at 'Sapienza' University in Rome) also for those participating in the DASP program.

In all patients, ZnT8 antibodies were measured as previously described using RIA (Medipan, Berlin, Germany) at San Raffaele Hospital in Milan (18).

\section{Genotyping}

The HLA-DRB1* and DQB1* loci typing was carried out by PCR followed by a reverse line blotting assay using an array of immobilized sequence-specific oligonucleotide probes (19). HLA genotypes were classified in three HLA risk categories based on the absolute risk values obtained in Italian population (20): i) high-risk genotypes: DRB $1^{*} 03$ DQB1*0201/DRB1*04-DQB1*0302 (DRB1*04 different from 0403$)$; ii) moderate-risk genotypes: DRB $1{ }^{*} 04$ DQB1*0302/DRB1*04-DQB1*0302, DRB1*03-DQB1*0201/ DRB1*03-DQB1*0201， DRB1*04-DQB1*0302/X, and DRB1*03/X (X different from DRB1*03, DRB1*04DQB1*0302 or $\left.\mathrm{DQB} 1{ }^{*} 0602 / 03\right)$; and iii) low-risk genotypes: all other genotypes.

\section{Follow-up study}

The drop out was $4 \%$ both in LADA and T2DM patients ( $n=8$ GADA positive and $n=18$ type 2 diabetes patients). The reasons of drop out were death, moved out of city, or adverse event (cancer). Therefore, 212 LADA patients and 412 type 2 diabetes were followed up for 7 years from the time of GADA screening to evaluate their progression toward insulin therapy.

In a subgroup of patients ( $n=16$ LADA and $n=20$ type 2 diabetes), GADA was re-tested at the time of starting insulin in order to evaluate the stability of positivity and titer.

Initiation of insulin therapy was part of a protocol treatment and the participating centers were blinded to the GADA results. Insulin requirement was defined as the clinical need to start insulin therapy in patients whose glycemic control, at each scheduled visit, became unacceptable (HbA1c $\geq 58 \mathrm{mmol} / \mathrm{mol}$ or $\geq 7.5 \%$ ) despite maximally tolerated combination of insulin-sensitizers (metformin and/or glitazones) and sulfonylurea therapy.

\section{Statistical analyses}

All analyses were performed using SAS, version 9.3. The frequencies were compared using the $\chi^{2}$ test, with Yates' continuity correction or Fisher's exact test. The ShapiroWilk test was used to test the normality of the distributions of continuous variables. For continuous variables, the difference between the mean value of the two groups was tested by the unpaired Student' $t$-test for normally distributed variables and the Mann-Whitney $U$ test for those which were not normally distributed. Kaplan-Meier curves were plotted and the log-rank test was performed to identify possible markers able to influence the progression to insulin requirement. The event time (insulin requirement) in the Kaplan-Meier curve was rounded to the nearest year interval. Cox regression analysis was performed to analyze the most important factors for future insulin requirement.

Multivariate logistic regression was performed to identify the possible predictors of the requirement of insulin. The investigated variables for the two models were as follows: GADA titer, number of antibodies, age at diagnosis, BMI, waist circumference, total cholesterol, HDL cholesterol, HbA1c, fasting glucose, and HLA DRB1*0301-DQB1*0201 haplotype positivity and diabetes treatment. A $P$ value $<0.05$ was considered statistically significant.

The study was approved by all local ethics committees (rif.491), and written informed consent was obtained from all patients.

\section{Results}

During the 7 years of follow-up, 119 (56.1\%) of 212 LADA patients and 86 (20.9\%) of 412 type 2 diabetes subjects required insulin therapy respectively.

In Table 1, we have given the clinical and biochemical characteristics of LADA patients subdivided according to their progression toward insulin requirement. LADA patients who progressed to insulin requirement were significantly younger, had a lower BMI and waist circumference and a higher HbA1c compared with those who did not progress; the median GADA titer was significantly higher in the group of subjects who progressed to insulin requirement. There was no significant 
Table 1 Clinical and biochemical characteristics of LADA patients at the time of GADA screening, subdivided according to insulin requirement during the 7 years of follow-up.

\begin{tabular}{|c|c|c|c|}
\hline & $\begin{array}{l}\text { Non-insulin } \\
\text { requirement }\end{array}$ & $\begin{array}{l}\text { Insulin } \\
\text { requirement }\end{array}$ & \\
\hline & Mean \pm S.D. & Mean \pm S.D. & $\boldsymbol{P}$ \\
\hline$n$ (male/female) & $48 / 45$ & $65 / 54$ & \\
\hline $\begin{array}{l}\text { Age at diagnosis } \\
\text { (years) }\end{array}$ & $54.5 \pm 10.8$ & $44.5 \pm 12.3$ & $<0.0001$ \\
\hline $\begin{array}{l}\text { Duration of disease } \\
\text { (months) }\end{array}$ & $30.7 \pm 20.2$ & $27.2 \pm 22.6$ & \\
\hline BMI $\left(\mathrm{kg} / \mathrm{m}^{2}\right)$ & $27.9 \pm 5.1$ & $25.7 \pm 4.2$ & 0.02 \\
\hline $\begin{array}{l}\text { Waist circumference } \\
(\mathrm{cm})\end{array}$ & $96.9 \pm 11.3$ & $90.6 \pm 10.8$ & 0.02 \\
\hline $\begin{array}{l}\text { Fasting glucose } \\
\quad(\mathrm{mg} / \mathrm{dl})\end{array}$ & $159.7 \pm 58.0$ & $170.0 \pm 53.9$ & \\
\hline $\mathrm{HbA} 1 \mathrm{c}(\mathrm{mmol} / \mathrm{mol})$ & $55.2 \pm 21.4$ & $62.8 \pm 23.5$ & 0.03 \\
\hline HbA1c (\%) & $7.2 \pm 1.9$ & $7.9 \pm 2.14$ & 0.03 \\
\hline $\begin{array}{l}\text { Total cholesterol } \\
\text { (mg/dl) }\end{array}$ & $215 \pm 49.2$ & $196.0 \pm 46.0$ & \\
\hline $\begin{array}{l}\text { HDL cholesterol } \\
(\mathrm{mg} / \mathrm{dl})\end{array}$ & $48.5 \pm 13.6$ & $50.3 \pm 14.3$ & \\
\hline Triglycerides (mg/dl) & $170.2 \pm 152.1$ & $135.2 \pm 77.6$ & \\
\hline Uric acid (mg/dl) & $5.2 \pm 1.4$ & $4.1 \pm 1.3$ & \\
\hline GADA titer ${ }^{a}$ & $11(7-72.4)$ & $88.9(7.3-100)$ & 0.01 \\
\hline $\begin{array}{l}\mathrm{DRB} 1 * 0301- \\
\mathrm{DQB} 1 * 0201^{\mathrm{b}}\end{array}$ & $27(29)$ & $57(47.9)$ & 0.008 \\
\hline
\end{tabular}

${ }^{a}$ Data are expressed as median (interquartile range)

${ }^{\mathrm{b}}$ Data are expressed as number and $(\%)$.

difference in HLA-DRB1 and HLA-DQB1 genotype distribution (high, moderate, or low) in the two groups of subjects; only the HLA DRB1*0301-DQB1*0201 haplotype frequency was significantly higher in the group of subjects who required insulin therapy earlier compared to the other group (47.9 vs $29 \%$ respectively, $P=0.008$ ).

Considering all subjects who required insulin (both LADA and type 2 diabetes patients), LADA subjects showed a significantly lower age at diagnosis, BMI, and waist circumference ( $P \leq 0.02$ for all comparisons), higher levels of fasting glucose, although not statistically significant, and higher levels of HbA1c and HDL cholesterol $(P=0.04$ and $P=0.03$ respectively) (data not shown) compared with type 2 diabetes patients.

Considering all subjects who did not require insulin, LADA patients showed a significantly higher levels of fasting glucose, HbA1c, and HDL cholesterol compared with type 2 diabetes subjects ( $P \leq 0.01$ for all comparisons); for the other parameters, there were no differences between the two groups of subjects (data not shown).

As illustrated in the Kaplan-Meier plots (Fig. 1A), patients with a significantly higher number of high GADA titer, 74/104 (71.1\%), required insulin during the 7 years of follow-up compared with those with low GADA titer
$45 / 108(41.6 \%)$ and to those with type 2 diabetes $86 / 412$ (20.9\%) ( $P<0.0001$, for both comparisons). Low GADA titer subjects showed, indeed, a faster progression to insulin requirement than type 2 diabetes subjects $\left(P<0.01\right.$; Fig. 1A). LADA patients with a BMI $\leq 25 \mathrm{~kg} / \mathrm{m}^{2}$ had a faster progression to insulin requirement than those with $\mathrm{BMI}>25 \mathrm{~kg} / \mathrm{m}^{2}$ (62 vs $48 \%$ respectively) $(P<0.0001$; Fig. 1B).

We also observed that positivity to both IA- $2_{\text {IC }}$ and ZnT8 ( $n=26$ subjects) was associated with a faster disease progression than that observed in subjects with both IA-2 IC and ZnT8 negativity (66 vs 48\% respectively) $(P<0.001$; Fig. 1C). The presence of ZnT8 antibodies showed a slight, but not significant, earlier requirement of insulin therapy $(P=0.06)$.

Figure 1D shows the Kaplan-Meier plots for LADA patients subdivided according to the treatment followed in the first year after diagnosis. The proportion of LADA patients who required insulin therapy was significantly higher in the group treated also with sulfonylurea in the first year after diagnosis than in those treated with diet and/or insulin sensitizers alone (either metformin and/ or glitazones; $P<0.001)$. Approximately $12 \%$ of LADA patients treated with diet and/or insulin sensitizers alone progressed to insulin requirement in the first 3 years of follow-up compared with $55 \%$ of LADA patients treated also with sulfonylurea during the first year after diagnosis $(P<0.01$; Fig. 1D)

At the time of starting insulin therapy, 13 out of 16 LADA patients maintained the same titer of GADA, whereas in the other three, all with low GADA titer at the first screening, the titer further decreased, although remaining above the limit of positivity; none of the 20 type 2 diabetes patients converted to GADA positivity.

Cox regression analysis showed that the most important factors associated with disease progression were: BMI $\leq 25 \mathrm{~kg} / \mathrm{m}^{2}(\mathrm{HR}=2.47 ; P<0.006)$ and high GADA titer $(\mathrm{HR}=3.42 ; \mathrm{P}<0.0003)$.

Multivariate logistic regression analysis of insulin requirement confirmed that only the presence of high GADA titer was a significant predictor of insulin requirement $(P<0.0001, \mathrm{OR}=6.95 ; \mathrm{CI}: 2.5-17.7)$. The presence of multiple antibodies was not a significant predictor $(P=0.12)$.

\section{Discussion}

This prospective study shows that $56.1 \%$ of LADA patients required insulin therapy during 7 years of follow-up compared with $20.9 \%$ of type 2 diabetes 

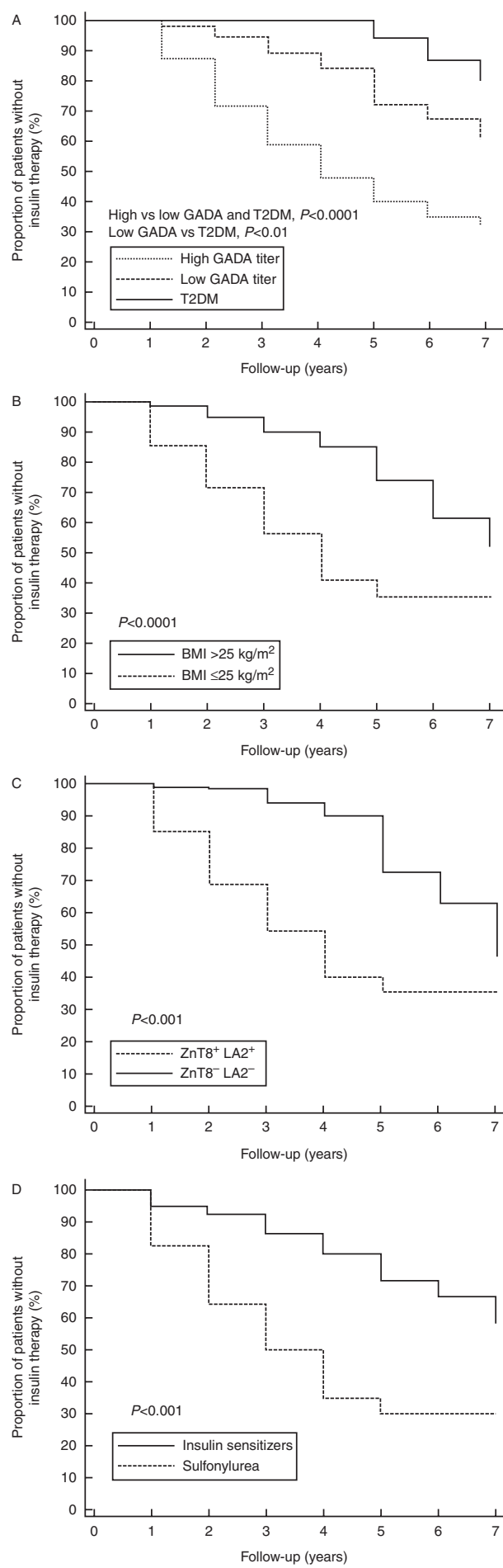

\section{Figure 1}

Kaplan-Meier plots of proportion of subjects, requiring insulin during 7-year follow-up, LADA with high or low GADA titer, and type 2 diabetes (A), Kaplan-Meier plots of proportion of LADA subjects subdivided according to BMI (B), according to IA-2IC and ZnT8 positivity (C), according to type of treatment (D). patients. This finding is in line with previous studies which report that a higher proportion of LADA subjects require insulin within a few years from diagnosis, compared with type 2 diabetes subjects $(21,22)$.

Brophy et al. (23) showed that in countries where the antibodies are assessed at the time of diagnosis, insulin therapy is initiated much earlier in LADA patients and this could be a bias in the comparison of different studies. In our study, the participating centers were not aware of antibodies positivity, and thus their decision to initiate insulin therapy was not influenced by this.

We have observed that the progression to insulin requirement is significantly higher and, above all, occur sooner in high GADA titer subjects compared with those with low GADA titer. These results underlined and reinforced our previous observations, showing that the presence of high GADA titer in LADA patients is associated with a more prominent trait indicative of insulin deficiency and consistent with a more severe profile of autoimmunity.

Following the first reports $(1,7,8)$, other studies have investigated whether GADA titer could influence the risk of progression to insulin therapy in LADA patients $(6,11,12$, $21,22,24,25,26)$. Some of these studies did not find a positive association between high GADA titer and risk of progression to insulin therapy; for example, differently from the UKPDS 25, the UKPDS 77 (12) found no association between GADA levels (classified according to thirds of the GADA level at 0.5 years) and insulin requirement, implying that GADA level was not associated with aggressiveness. In a recent cross-sectional study, the Action LADA 7 (27) high GADA titer patients were compared with low GADA titer patients in terms of time to insulin requirement, but no statistical significant difference was found. However, in this case, type 1 diabetes and LADA patients were considered as a single group and the LADA group was not evaluated separately. Another consistent group of studies found that high titer of GADA was associated with a shorter insulin-free period $(9,21,25)$.

Our data, evaluated by Kaplan-Meier plots, suggest that GADA titer, rather than its presence, could play an important role in determining the risk of progression to insulin requirement. Multiple regression analysis further extends these findings showing that among the factors we investigated, high GADA titer was found to be the best predictor of progression to insulin requirement.

In the light of the previous considerations, our study definitely adds some arguments in favor of the role played by the high GADA titer in increasing the risk of insulin requirement in LADA: different from most of the other studies, which were cross-sectional, this study is a 
prospective one and is based on a high sensitivity and specificity assay to detect GADA.

Furthermore, we compared the insulin requirement between the two distinct populations we obtained from a previous evidence of a bimodal distribution of GADA titers (low and high GADA) in LADA patients.

When we retrospectively evaluated the $\mathrm{HbA1c}$ values of patients at baseline, we noted a significant difference between patients who progressed and did not progress to insulin requirement (Table 1). However, in the KaplanMeyer analysis, when we subdivided patients according to different variables (GADA titer, BMI, ZnT8/IA-2 positivity, and type of treatment) we did not observe any significant difference at baseline between the groups as far as $\mathrm{HbA1c}$ is concern.

$\beta$-cell secretion could have been impaired in the early phase of the disease in patients with higher HbA1c at baseline (Table 1). This could have influenced the frequency of insulin treatment during the course of the study. The influence of prior $\beta$-cell damage vis-à-vis continued autoimmune destruction of $\beta$ cells cannot be conclusively determined from our data.

The stability of GADA levels and titers have been investigated in LADA patients $(2,12)$. Desai et al. (12) found that GADA levels varied between 0.5 and 6 years after diagnosis in some patients, but those with higher values at 0.5 years, tended to remain with a high titer at 3 and 6 years, whereas in those with low values at 0.5 years remained low thereafter. Borg et al. (28) also found that in LADA patients, GADA titer persisted for up to 12 years. In this study, some samples of LADA patients were retested for GADA at the time of starting insulin therapy: the majority maintained the same titer of GADA. We also observed that none of the type 2 diabetes patients converted to GADA positivity. In view of these results we could hypothesize that GADA is persistent over time.

Some evidence has previously demonstrated that the presence of multiple autoantibodies rather than high GADA titer could predict insulin requirement in LADA subjects: Bottazzo et al. (2) found that in type 2 diabetes patients, the presence of IA-2A in addition to GADA increased the likelihood of insulin therapy within 6 years from diagnosis; more recently Maioli et al. (6) found that the number of antibodies was more important than high titers of GADA for predicting insulin dependence.

However, we observed that high GADA titer is associated with faster insulin progression during a 7 years of follow-up regardless of the number of antibodies and that the presence of multiple antibodies is not a significant predictor of insulin requirement.
Although according to our multivariate analysis, high GADA titer seems to be the best predictor of progression to insulin dependence, Kaplan-Meier plots demonstrate that positivity for both IA-2 $2_{\text {IC }}$ and ZnT8 increased the risk of progression to insulin therapy. Kawasaki et al. (29) found that the presence of ZnT8 and/or IA-2 $2_{\text {IC }}$ increases the progression to insulin requirement in LADA patients. We showed that HLA DRB1*0301-DQB1*0201 haplotype frequency was significantly higher in subjects requiring insulin, which is consistent with the observation that patients with high GADA titer have the highest frequency of DRB1*03-DQB1*0201 (50\%) (13). LADA patients with a BMI $\leq 25 \mathrm{~kg} / \mathrm{m}^{2}$ have a faster progression to insulin requirement than those with a BMI $>25 \mathrm{~kg} / \mathrm{m}^{2}$; this is in line with previous studies $(6,24,30,31)$.

In this study, we show that insulin sensitizers maintained the insulin-free period longer than sulfonylurea. This could be either due to the increased $\beta$-cell apoptosis from use of sulfonylurea $(32,33)$ or due to the protective effect of insulin sensitizers (34).

When we analyzed BMI and HbA1c levels in patients subdivided into two groups depending on prescribed sulfonylureaor insulin sensitizer, we observed that there were no statistical significant differences for these parameters among the two groups $(P<0.05$ for all), thus we would hypothesize that the type of treatment may influence the progression toward insulin requirement.

There are no current guidelines for LADA treatment at present; however some beneficial effects of insulin have been reported. The Tokyo study has showed improved maintenance of the serum $\Sigma$-C-peptide over 5 years in the group receiving insulin compared with sulfonylurea (35). The subgroup analysis suggested that patients with high GADA titers and preserved C-peptide at baseline were less likely to progress to the insulin-dependent stage with early administration of small doses of insulin. These observations reinforce the importance of the identification of LADA patients with high GADA titer and the administration of early insulin therapy in order to preserve $\beta$-cell function for a longer time (Supplementary Tables 1 and 2, see section on supplementary data given at the end of this article).

Supplementary data

This is linked to the online version of the paper at http://dx.doi.org/10.1530/ EJE-14-0342.

\section{Declaration of interest}

The authors declare that there is no conflict of interest that could be perceived as prejudicing the impartiality of the research reported. 
Funding

This study is sponsored by 'Fondazione Diabete e Ricerca' (ONLUS) of Società Italiana di Diabetologia and funded by an unconditional grant from Novo Nordisk, Italy.

\section{Author contribution statement}

S Zampetti wrote the manuscript; G Campagna and J Osborn performed data analysis; C Tiberti and E Bosi responsible for antibody testing, M Spoletini, M L Arpi, G De Simone, E Cossu, L Cocco, and F Giorgino researched data and reviewed the manuscript; $M$ Spoletini performed the genetic typing and reviewed the manuscript; and R Buzzetti implemented the study design and wrote the manuscript. All authors approved the final version of the manuscript.

\section{Acknowledgements}

NIRAD follow-up investigators: G Adda and S Di Lembo, S Giuseppe Ospedale (Milano); A. Aglialoro and A. Cattaneo, Villa Scassi (Genova); R. Anichini, Presidio Ospedaliero (Pistoia); A. Arcangeli, ASL 4 (Prato); M.L. Arpi, Endocrinologia, Ospedale Garibaldi Nesima (Catania); R.G. Bardini, Università (Firenze); P. Basciano, Ospedale Sant'Antonio Abate Erice (Trapani); M. Bracaccia and S. Pistoni, Ospedale S. Maria della Stella di Orvieto (Terni); D. Bracaglia, Centro Diabetologico ASL Roma B (Roma); V. Borzì, Ospedale Vittorio Emanuele II (Catania); F. Cannatà, Ospedale S. Maria della Croci (Ravenna); G. Capitano, ASL 2 (Salerno); M. Cignarelli and S. Piemontese, Università (Foggia), G. Capuano, Centro Diabetologico (Salerno); S. Carro, Centro Antidiabetico ASL 5 (La Spezia); C. Cazzalini, Ospedale Maggiore (Crema); L. Cocco, Diabetologia e Malattie Metaboliche, Ospedale Cardarelli, (Campobasso); A.M. Ciccarone, Dipartimento di Endocrinologia e Metabolismo Ospedale di Cisanello (Pisa); G. Cicioni, ASL 4 (Terni); E. Cossu and $F$. Sano, Centro Diabetologico e Malattie Metaboliche, Policlinico Monserrato (Cagliari); D. Cucinotta and A. Di Benedetto, Policlinico Universitario G. Martino (Messina); G. De Mattia and M.R. Mollica, Policlinico Umberto I (Roma); S. De Cosmo and A. Minenna, Casa Sollievo della Sofferenza San Giovanni Rotondo (Foggia); A. Dei Cas, Dipartimento di Medicina Interna, Università degli Studi di Parma (Parma); G. De Simone, ASL Napoli 3, San Giorgio a Cremano (Napoli); P. Di Berardino, Ospedale di Atri (Teramo); F. Dotta and V. Contini, Policlinico Umberto I (Roma); I. Franzetti, Ospedale di Circolo (Varese); S. Frontoni and D. Bracaglia, Università'Tor Vergata' (Roma); G. Gadaleta, Presidio Ospedaliero Cittiglio (Varese); G. Galeone and A.V. Magiar, Ospedale di Pescia (Pistoia); M.R. Garofano, Ospedale Cannizzaro Aci Castello (Catania); S. Gentile and G. Guarino, Dipartimento Medicina Clinica e Sperimentale, Secondo Policlinico Napoli (Napoli); R. Giansanti, INRCA (Ancona); S. Genovese, Istituto Clinico Humanitas Rozzano (Milano); A. Giancaterini, INRCA (Roma); S. Leotta, Ospedale Sandro Pertini (Roma); E. Gianni and S. Burrafato, Ospedale M.P. Arezzo (Ragusa), C. Giordano, Endocrinologia e Malattie Metaboliche, Università degli Studi di Palermo (Palermo); A. Gigante and A. Cicalò, Ospedale S. Francesco (Nuoro); F. Giorgino, Endocrinologia, Policlinico di Bari (Bari); A. Gnasso and E. Fiaschi, Policlinico Mater Domini (Catanzaro); G. Grossi and F. Deverardinis, ASL1 di Paola (Cosenza); L. lanni, ASL 4 (Prato); C. lovine, Policlinico Federico II (Napoli); R. Lauro, M. Federici and V. Spallone, Università di Tor Vergata (Roma), A. Lo Presti and A.M. Scarpetta, Ospedale S. Biagio Marsala (Trapani); R. Lunari, Ospedale Maggiore (Parma); R. Manna and A. Margotta, Presidio Ospedaliero di Tradate (Varese), E. Mantovani, Centro Antidiabetico, Azienda Ospedaliera C. Poma (Mantova); M.C. Matteoli, Ospedale Bambino Gesù (Roma); E. Matteucci and F. Chiesi, Azienda Ospedaliera (Pisa); A. Maran, Università (Padova); P. Mascetti and T. Quintana, Azienda Ospedaliera S. Anna (Como); P. Mazzucca, Ospedale degli Infermi (Rimini); P. Melga and R. Cordera, DISEM (Genova); I. Meloncelli, Centro Diabetologico Ospedale Civile Madonna del Soccorso, (Ascoli Piceno); S. Morano, Dipartimento di Medicina Interna e Specialità Mediche, Università'Sapienza' (Roma),
L. Morviducci, Diabetologia, Ospedale San Carlo Forlanini, (Roma), M. Nannipieri, Università (Pisa); M. Parillo, Ospedale (Caserta); A. Pacifico, Università (Sassari); G. Pascal, Ospedale di Suzzara (Mantova); E. Papini and F. Graziano, Ospedale Regina Apostulorum Albano Laziale (Roma); A. Passaro, Università (Ferrara); P. Pata, Ospedale Piemonte (Messina); M. Poli, Ospedale S. Biagio di Bovolone (Verona); A.E. Pontiroli, Ospedale San Paolo, (Milano), P. Pozzilli, L. Cipolloni and C. Guglielmi, Campus BioMedico, (Roma); L. Puccio, Ospedale Civile (Catanzaro); L.M. Raffa, USL 1 (Sanremo); D. Richini, Ospedale di Vallecamonica (Brescia); R. Romano, Presidio Ospedaliero dell'Annunziata (Cosenza); C. Rotella, Azienda Ospedaliera, Careggi, (Firenze); G. Santantonio, Ospedale San Paolo di Civitavecchia (Roma); M.S. Sbriglia, Ospedale SS. Annunziata Savigliano (Cuneo); M. Songini, V. Cau, Ospedale S. Michele, (Cagliari); A. Scorsone, Ospedale (Palermo); V. Spallone, Dipartimento di Medicina Interna, Università'Tor Vergata', (Roma); C. Taboga, Ospedale Civile (Udine); P. Tatti, Ospedale San Giuseppe, Marino, (Roma), A. Turco, Ospedale Carlo Poma di Asola (Cremona); M. Trovati and E. Fiori, Ospedale San Luigi di Orbassano (Torino); M. Vasta, ASL 2 (Urbino); F. Vitale, Ospedale San Carlo (Potenza); D. Zavaroni, AUSL (Piacenza).

Steering Committee. Raffaella Buzzetti (chair), Emanuele Bosi, Efisio Cossu, Francesco Dotta, Stefano Genovese, Andrea Giaccari, Carla Giordano, Francesco Giorgino, Marco Songini.

Executive Committee. Sergio Di Pietro, Concetta Suraci, Mattia Locatelli. Laboratories. Autoantibodies: Milan, San Raffaele Hospital: Emanuele Bosi, Elena Bazzigaluppi; Perugia University: Alberto Falorni; Rome, 'Sapienza' Università: Claudio Tiberti; Genetics: Rome, 'Sapienza' University: Marco Capizzi, Lidia Marandola, Antonio Petrone.

\section{References}

1 Turner R, Stratton I, Horton V, Manley S, Zimmet P, Mackay IR, Shattock M, Bottazzo GF \& Holman R. UKPDS 25: autoantibodies to isletcell cytoplasm and glutamic acid decarboxylase for prediction of insulin requirement in type 2 diabetes: UK Prospective Diabetes Study Group. Lancet 1997350 1288-1293. (doi:10.1016/S0140-6736(97)03062-6)

2 Bottazzo GF, Bosi E, Cull CA, Bonifacio E, Locatelli M, Zimmet P, Mackay IR \& Holman RR. IA-2 antibody prevalence and risk assessment of early insulin requirement in subjects presenting with type 2 diabetes (UKPDS 71). Diabetologia 200548 703-708. (doi:10.1007/s00125-005-1691-9)

3 Trabucchi A, Faccinetti NI, Guerra LL, Puchulu FM, Frechtel GD, Poskus E \& Valdez SN. Detection and characterization of ZnT8 autoantibodies could help to screen latent autoimmune diabetes in adult-onset patients with type 2 phenotype. Autoimmunity 201245 137-142. (doi:10.3109/08916934.2011.604658)

4 Tuomi T, Groop LC, Zimmet PZ, Rowley MJ, Knowles W \& Mackay IR. Antibodies to glutamic acid decarboxylase reveal latent autoimmune diabetes mellitus in adults with a non-insulin-dependent onset of disease. Diabetes 199342 359-362. (doi:10.2337/diab.42.2.359)

5 Akesson C, Uvebrant K, Oderup C, Lynch K, Harris RA, Lernmark A, Agardh CD \& Cilio CM. Altered natural killer (NK) cell frequency and phenotype in latent autoimmune diabetes in adults (LADA) prior to insulin deficiency. Clinical and Experimental Immunology 2010 161 48-56. (doi:10.1111/j.1365-2249.2010.04114.x)

6 Maioli M, Pes GM, Delitala G, Puddu L, Falorni A, Tolu F, Lampis R, Orrù V, Secchi G, Cicalò AM et al. Number of autoantibodies and HLA genotype, more than high titers of glutamic acid decarboxylase autoantibodies, predict insulin dependence in latent autoimmune diabetes of adults. European Journal of Endocrinology 2010163 541-549. (doi:10.1530/EJE-10-0427)

7 Gottsäter A, Landin-Olsson M, Lernmark A, Fernlund P, Sundkvist G \& Hagopian WA. Glutamate decarboxylase antibody levels predict rate of $\beta$-cell decline in adult-onset diabetes. Diabetes Research and Clinical Practice 199527 133-140. 
8 Niskanen LK, Tuomi T, Karjalainen J, Groop LC \& Uusitupa MI. GAD antibodies in NIDDM. Ten-year follow-up from the diagnosis. Diabetes Care 199518 1557-1565. (doi:10.2337/diacare.18.12.1557)

9 Radtke MA, Midthjell K, Nilsen TI \& Grill V. Heterogeneity of patients with latent autoimmune diabetes in adults: linkage to autoimmunity is apparent only in those with perceived need for insulin treatment: results from the Nord-Trøndelag Health (HUNT) study. Diabetes Care 200932 245-250. (doi:10.2337/dc08-1468)

10 Genovese S, Bazzigaluppi E, Gonçalves D, Ciucci A, Cavallo MG, Purrello F, Anello M, Rotella CM, Bardini G, Vaccaro O et al. Clinical phenotype and $\beta$-cell autoimmunity in Italian patients with adult-onset diabetes. European Journal of Endocrinology $2006 \mathbf{1 5 4}$ 441-447. (doi:10.1530/eje.1.02115)

11 Maruyama T, Nakagawa T, Kasuga A \& Murata M. Heterogeneity among patients with latent autoimmune diabetes in adults. Diabetes/Metabolism Research and Reviews 201127 971-974. (doi:10.1002/dmrr.1238)

12 Desai M, Cull CA, Horton VA, Christie MR, Bonifacio E, Lampasona V, Bingley PJ, Levy JC, Mackay IR, Zimmet P et al. GAD autoantibodies and epitope reactivities persist after diagnosis in latent autoimmune diabetes in adults but do not predict disease progression: UKPDS 77. Diabetologia 200750 2052-2060. (doi:10.1007/s00125-007-0745-6)

13 Buzzetti R, Di Pietro S, Giaccari A, Petrone A, Locatelli M, Suraci C, Capizzi M, Arpi ML, Bazzigaluppi E, Dotta F et al. High titer of autoantibodies to GAD identifies a specific phenotype of adult-onset autoimmune diabetes. Diabetes Care 200730 932-938. (doi:10.2337/dc06-1696)

14 Zampetti S, Capizzi M, Spoletini M, Campagna G, Leto G, Cipolloni L, Tiberti C, Bosi E, Falorni A, Buzzetti R et al. GADA titer-related risk for organ-specific autoimmunity in LADA subjects subdivided according to gender (NIRAD study 6). Journal of Clinical Endocrinology and Metabolism 201297 3759-3765. (doi:10.1210/jc.2012-2037)

15 American Diabetes Association. Diagnosis and classification of diabetes mellitus. Diabetes Care 200629 S43-S48.

16 Bonifacio E, Genovese S, Braghi S, Bazzigaluppi E, Lampasona V, Bingley PJ, Rogge L, Pastore MR, Bognetti E, Bottazzo GF et al. Islet autoantibody markers in insulin dependent diabetes: risk assessment strategies yielding high sensitivity. Diabetologia 199538 816-822. (doi:10.1007/s001250050358)

17 Bingley PJ, Bonifacio E \& Mueller PW. Diabetes antibody standardization program: first assay proficiency evaluation. Diabetes $2003 \mathbf{5 2}$ 1128-1136. (doi:10.2337/diabetes.52.5.1128)

18 Lampasona V, Petrone A, Tiberti C, Capizzi M, Spoletini M, Di Pietro S, Songini M, Bonicchio S, Giorgino F, Bonifacio E et al. Zinc transporter 8 antibodies complement GAD and IA-2 antibodies in the identification and characterization of adult-onset autoimmune diabetes. Non-insulin requiring autoimmune diabetes (NIRAD) 4. Diabetes Care 201033 104-108. (doi:10.2337/dc08-2305)

19 Buzzetti R, Galgani A, Petrone A, Del Buono ML, Erlich HA, Bugawan TL, Lorini R, Meschi F, Multari G, Pozzilli P et al. Genetic prediction of type 1 diabetes in a population with low frequency of HLA risk genotypes and low incidence of the disease (the DIABFIN study). Diabetes/Metabolism Research and Reviews 20042 137-143. (doi:10.1002/dmrr.426)

20 Petrone A, Bugawan TL, Mesturino CA, Nisticò L, Galgani A, Giorgi G, Cascino I, Erlich HA, Di Mario U \& Buzzetti R. The distribution of HLA class II susceptible/protective haplotypes could partially explain the low incidence of type 1 diabetes in continental Italy (Lazio region). Tissue Antigens 200158 385-394. (doi:10.1034/j.1399-0039.2001.580607.x)

21 Lee SA, Lee WJ, Kim EH, Yu JH, Jung CH, Koh EH, Kim MS, Park JY \& Lee KU. Progression to insulin deficiency in Korean patients with type 2 diabetes mellitus positive for anti-GAD antibody. Diabetic Medicine 201128 319-324. (doi:10.1111/j.1464-5491.2011.03241.x)

22 Davis TM, Zimmet P, Davis WA, Bruce DG, Fida S \& Mackay IR. Autoantibodies to glutamic acid decarboxylase in diabetic patients from a multi-ethnic Australian community: the Fremantle diabetes study. Diabetic Medicine 200017 667-674. (doi:10.1046/j.1464-5491.2000.00359.x)

23 Brophy S, Yderstraede K, Mauricio D, Hunter S, Hawa M, Pozzilli P, Schernthaner G, Schloot N, Buzzetti R, Davies H et al. Time to insulin initiation cannot be used in defining latent autoimmune diabetes in adults. Diabetes Care 200831 439-441. (doi:10.2337/dc07-1308)

24 Falorni A \& Brozzetti A. Diabetes-related antibodies in adult diabetic patients. Best Practice \& Research. Clinical Endocrinology \& Metabolism 200519 119-133. (doi:10.1016/j.beem.2004.11.010)

25 Kasuga A, Maruyama T, Nakamoto S, Ozawa Y, Suzuki Y \& Saruta T. High titer autoantibodies against glutamic acid decarboxylase plus autoantibodies against insulin and IA-2 predicts insulin requirement in adult diabetic patients. Journal of Autoimmunity 199912 131-135. (doi:10.1006/jaut.1998.0261)

26 Takeda H, Kawasaki E, Shimizu I, Konoue E, Fujiyama M, Murao S, Tanaka K, Mori K, Tarumi Y, Seto I et al. Clinical, autoimmune, and genetic characteristics of adult-onset diabetic patients with GAD autoantibodies in Japan (Ehime Study). Diabetes Care 200225 995-1001. (doi:10.2337/diacare.25.6.995)

27 Hawa MI, Kolb H, Schloot N, Beyan H, Paschou SA, Buzzetti R, Mauricio D, De Leiva A, Yderstraede K, Beck-Neilsen H et al. Adult-onset autoimmune diabetes in Europe is prevalent with a broad clinical phenotype: Action LADA 7. Diabetes Care 201336 908-913. (doi:10.2337/dc12-0931)

28 Borg H, Gottsäter A, Fernlund P \& Sundkvist G. A 12-year prospective study of the relationship between islet antibodies and $\beta$-cell function at and after the diagnosis in patients with adult-onset diabetes. Diabetes 200251 1754-1762. (doi:10.2337/diabetes.51.6.1754)

29 Kawasaki E, Nakamura K, Kuriya G, Satoh T, Kuwahara H, Kobayashi M, Abiru N, Yamasaki H \& Eguchi K. Autoantibodies to insulin, insulinoma-associated antigen-2, and zinc transporter 8 improve the prediction of early insulin requirement in adult-onset autoimmune diabetes. Journal of Clinical Endocrinology and Metabolism 201095 707-713. (doi:10.1210/jc.2009-1733)

30 Lohmann T, Kellner K, Verlohren HJ, Krug J, Steindorf J, Scherbaum WA $\&$ Seissler J. Titre and combination of ICA and autoantibodies to glutamic acid decarboxylase discriminate two clinically distinct types of latent autoimmune diabetes in adults (LADA). Diabetologia $2001 \mathbf{4 4}$ 1005-1010. (doi:10.1007/s001250100602)

31 Li X, Huang G, Yan X, Yang L \& Zhou ZG. Relation between insulin resistance and glutamic acid decarboxylase antibody titers in latent autoimmune diabetes in adults. J Nan Fang Yi Ke Da Xие Xие Bao 2010 30 1247-1249.

32 Cernea S, Buzzetti R \& Pozzilli P. $\beta$-cell protection and therapy for latent autoimmune diabetes in adults. Diabetes Care 200932 (Suppl 2) S246-S252. (doi:10.2337/dc09-S317)

33 Maedler K, Carr RD, Bosco D, Zuellig RA, Berney T \& Donath MY. Sulfonylurea induced $\beta$-cell apoptosis in cultured human islets. Journal of Clinical Endocrinology and Metabolism 200590 501-506. (doi:10.1210/jc.2004-0699)

34 Yang Z, Zhou Z, Li X, Huang G \& Lin J. Rosiglitazone preserves islet $\beta$-cell function of adult-onset latent autoimmune diabetes in 3 years follow-up study. Diabetes Research and Clinical Practice 200983 54-60. (doi:10.1016/j.diabres.2008.09.044)

35 Maruyama T, Shimada A, Kanatsuka A, Kasuga A, Takei I, Yokoyama J \& Kobayashi T. Multicenter prevention trial of slowly progressive type 1 diabetes with small dose of insulin (the Tokyo study): preliminary report. Annals of New York Academy of Sciences 20031005 362-369. (doi:10.1196/annals.1288.060)

Received 28 April 2014

Revised version received 3 September 2014

Accepted 11 September 2014 